\title{
Development of a Refined Quarter Car Model for the Analysis of Discomfort due to Vibration
}

\author{
A. N. Thite \\ Department of Mechanical Engineering and Mathematical Sciences, Faculty of Technology, Design and Environment, \\ Oxford Brookes University, Wheatley, Oxford OX33 1HX, UK \\ Correspondence should be addressed to A. N. Thite, athite@brookes.ac.uk
}

Received 24 April 2012; Accepted 26 May 2012

Academic Editor: Joseph C. S. Lai

Copyright ( 92012 A. N. Thite. This is an open access article distributed under the Creative Commons Attribution License, which permits unrestricted use, distribution, and reproduction in any medium, provided the original work is properly cited.

In the automotive industry, numerous expensive and time-consuming trials are used to "optimize" the ride and handling performance. Ideally, a reliable virtual prototype is a solution. The practical usage of a model is linked and restricted by the model complexity and reliability. The object of this study is development and analysis of a refined quarter car suspension model, which includes the effect of series stiffness, to estimate the response at higher frequencies; resulting Maxwell's model representation does not allow straightforward calculation of performance parameters. Governing equations of motion are manipulated to calculate the effective stiffness and damping values. State space model is arranged in a novel form to find eigenvalues, which is a unique contribution. Analysis shows the influence of suspension damping and series stiffness on natural frequencies and regions of reduced vibration response. Increase in the suspension damping coefficient beyond optimum values was found to reduce the modal damping and increase the natural frequencies. Instead of carrying out trial simulations during performance optimization for human comfort, an expression is developed for corresponding suspension damping coefficient. The analysis clearly shows the influence of the series stiffness on suspension dynamics and necessity to incorporate the model in performance predictions.

\section{Introduction}

In the vehicle suspension design, dependent on the usage pattern, handling and ride comfort performance have contradicting requirements [1]. The parameter range for optimum suspension design can vary for different vehicles; often complex combinations of parameters form a solution. In the industry, numerous expensive and time-consuming experimental trials are used to "optimize" the performance. Ideally, a reliable virtual prototype is a solution. The practical usage of a vehicle model is linked and restricted by model complexity; a refined model without complexities is a requirement. One of the modelling difficulties is the presence of various compliances and their connections within the vehicle suspension system. The object of this research is the investigation of a quarter car model with the aim of analysing comfort considering the compliances in shock absorber and suspension top mount; there is series stiffness in the model.

Models of varying complexity, considering only linear dynamic elements to systems involving nonlinear elements, have been developed to predict, specifically, vehicle handling and, to some extent, ride comfort. Shock absorbers have been modelled to represent hydromechanical behaviour for example, $[2,3]$ the complexities do not allow easy integration of the model into vehicle dynamics applications. It is difficult to perform parametric studies and visualize the outcome. In passive simplified models, shock absorber is treated as a viscous damper, simplest being a linear model. Often, elements connecting the shock absorbers to the suspension system and the effects of various noise and vibration designs are ignored $[4,5]$.

Simplest vehicle model used to assess discomfort due to vibration is of two degrees of freedom (DOF) [1], commonly known as a quarter car model. Only vertical motion is considered for discomfort quantification [6]. In the model, tyre is represented by a stiffness, wheel and associated elements are represented by a mass, and suspension is represented by a spring and a damper working in parallel (Kelvin-Voigt model, hence forth called as an ordinary model) and vehicle body by a mass. The representation is very simplistic and 
may not capture the dynamic response accurately; impulsive forces would make suspension spring and damper combination act practically like a rigid element. In the industry, to eliminate adverse force transmission, a stiffness in series (generally called the top mount) to the shock absorber and spring combination is introduced; the presence of series stiffness also benefits noise, vibration, and harshness (NVH) performance at higher frequencies, above about $100 \mathrm{~Hz}$. However, introduction of the series stiffness may have a complex influence at lower frequencies, below about $30 \mathrm{~Hz}$, affecting vehicle ride comfort. Further, compliance within the shock absorber is not considered in the Kelvin-Voigt model. To an extent, these compliant elements limit the damping force by relieving the pressure inside shock absorber. Overall, an appropriate representation of series stiffness requires the use of the Maxwell model or a viscoelastic element.

The series stiffness, as in 1 DOF model, may influence effective damping; two modal damping ratios of the quarter car model can be different from those based on the KelvinVoigt model and the variations can be a complex function of the suspension damping coefficient and the stiffness. Further, the effect of series stiffness may not be equal on the modes of vibration. The resonance frequencies are expected to be dependent on the damping coefficient. For optimization, sufficient damping is required for both modes. The modal damping ratio calculation is not straightforward; in practice, they are not calculated explicitly; instead cost functions are formed and trials are carried out to reduce the vibratory responses directly. The process does not provide insight into the complex problem. It is desirable to find a combination of parameters resulting in large damping ratios and small shift in resonance frequency, without having to perform numerous forced vibration analyses; the focus of optimization continues to be the response reduction but achieved through an alternative approach. Availability of an explicit formula for effective damping can minimize the optimization effort and provide critical information.

In what follows, the quarter car model dynamic response with and without series stiffness is compared to show the effect of series stiffness. For the harmonic input, equations of motion are rearranged to calculate effective stiffness and damping values. Using the knowledge of forces transmitted through the series stiffness, a novel form of state space equations is generated so as to calculate the natural frequencies and modal damping ratios. The effects of combination of suspension damping coefficient and series stiffness are analysed showing regions of reduced vibration response. A simplified expression is developed for optimum suspension damping with the aim of mainly reducing wheel hop frequency response; the wheel hop frequency response will be shown being more sensitive to the presence of series stiffness than the vehicle body mode response. The model clearly shows the influence of series stiffness on the modal damping ratios and the natural frequencies.

\section{Vehicle Dynamic Lumped Parameter Model}

The influence of damping on shift in the resonance frequency and variation in the corresponding amplitude for 1 DOF

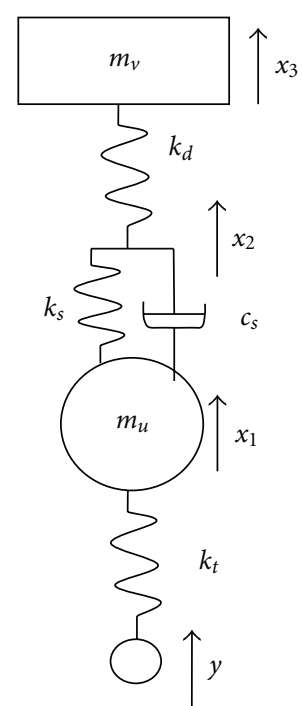

Figure 1: Schematic of a quarter car model with the use of Maxwell's model to represent the suspension dynamics.

Maxwell's model is well documented. The vehicle suspension system with series stiffness may show similar influence, but the influence may not be a simple function of the damping value alone; relative values of series and the tyre stiffnesses may play a significant role. Further, the behaviour due to different forms of input may also be complex.

Initially, in the next section two models of the suspension system, with and without series stiffness, are compared for their effect on the steady-state frequency domain response. Later, a state space model is used to calculate the natural frequencies and the modal damping ratios. An expression is developed for optimum damping. In the model, stiffness and damping coefficients used are linear equivalent parameters.

\section{Application of Maxwell's Model to Represent the Quarter Car Dynamics}

Figure 1 shows schematic of Maxwell's model representing the corner of a vehicle. The equations of motion can be obtained by applying Newton's 2nd law, which after some manipulations are given by

$$
\begin{gathered}
m_{v} \ddot{x}_{3}+k_{d} x_{3}-k_{d} x_{2}=0, \\
c_{s} \dot{x}_{1}-c_{s} \dot{x}_{2}+k_{s} x_{1}-k_{s} x_{2}-k_{d} x_{2}+k_{d} x_{3}=0, \\
m_{u} \ddot{x}_{1}+c_{s}\left(\dot{x}_{1}-\dot{x}_{2}\right)+k_{s}\left(x_{1}-x_{2}\right)+k_{t} x_{1}=k_{t} y .
\end{gathered}
$$

There is an additional response variable $x_{2}$ compared to an ordinary 2 DOF model. For harmonic excitation, this variable can be eliminated from the analysis by substituting equivalent values in terms of response variables $x_{1}$ and $x_{3}$, reducing the system to 2 DOFs. Hence, for forced vibration analysis, we have

$$
\left(-\mathbf{M} \omega^{2}+j \omega \mathbf{C}+\mathbf{K}\right) \mathbf{X}=\mathbf{F},
$$


where mass matrix is given by

$$
\mathbf{M}=\left[\begin{array}{cc}
m_{u} & 0 \\
0 & m_{v}
\end{array}\right],
$$

effective damping matrix is given by

$$
\mathbf{C}=\left[\begin{array}{cc}
\frac{k_{d}^{2} c_{s}}{\left(\left(k_{d}+k_{s}\right)^{2}+c_{s}^{2} \omega^{2}\right)} & \frac{-k_{d}^{2} c_{s}}{\left(\left(k_{d}+k_{s}\right)^{2}+c_{s}^{2} \omega^{2}\right)} \\
\frac{-k_{d}^{2} c_{s}}{\left(\left(k_{d}+k_{s}\right)^{2}+c_{s}^{2} \omega^{2}\right)} & \frac{k_{d}^{2} c_{s}}{\left(\left(k_{d}+k_{s}\right)^{2}+c_{s}^{2} \omega^{2}\right)}
\end{array}\right],
$$

and effective stiffness matrix is given by

$$
\mathbf{K}=\left[\begin{array}{cc}
k_{t}+\frac{\left(c_{s}^{2} \omega^{2} k_{d}+k_{d} k_{s}\left(k_{d}+k_{s}\right)\right)}{\left(\left(k_{d}+k_{s}\right)^{2}+c_{s}^{2} \omega^{2}\right)} & -k_{d} \\
\frac{-k_{d}\left(c_{s}^{2} \omega^{2}+k_{s}\left(k_{d}+k_{s}\right)\right)}{\left(\left(k_{d}+k_{s}\right)^{2}+c_{s}^{2} \omega^{2}\right)} & k_{d}-\frac{k_{d}^{2}\left(k_{d}+k_{s}\right)}{\left(\left(k_{d}+k_{s}\right)^{2}+c_{s}^{2} \omega^{2}\right)}
\end{array}\right] .
$$

The elements of matrices are as follows: $m_{u}$ is the effective mass of wheel hub, $m_{v}$ is a quarter of vehicle body mass, $c_{s}$ is the suspension damping coefficient, $k_{s}$ is the suspension stiffness, $k_{t}$ is the tyre stiffness, $k_{d}$ is the series stiffness, and $\omega$ is the excitation frequency. The stiffness matrix, $\mathbf{K}$, has nonlinear elements; it is now dependent on the damping coefficient and excitation frequency. In the suspension system, for effective isolation at higher frequencies, the series spring stiffness is likely to be of the order of tyre stiffness [7] for a range of displacements; the effect on second resonance (the wheel hop frequency) is expected to be significant. The relative values of stiffness and damping coefficient determine the resonance frequency sensitivity. If the series stiffness is relatively large, the system would act as an ordinary two-DOF model and the spring and the damper being connected in parallel, irrespective of damping in the system.

The effective damping (6) is also a complex function of series stiffness, suspension damping coefficient, and frequency, showing nonlinear behaviour. For large series stiffness values, it tends to the suspension damping coefficient. On the other hand, for small values, the damping is a complex function of series stiffness and frequency. The effective value determines the response peaks of wheel hub and vehicle body motion. Later in this paper an approximate expression is developed to estimate the optimum value of suspension damping coefficient.

Using (4), for a harmonic input, vehicle body and wheel hub responses can be calculated. The parameters used for the calculation are listed in Table 1, which can be obtained, for example, using the methods of [8]. Figure 2(a) shows wheel hub motion; there are two peaks, one each for the vehicle body bounce mode (around $2 \mathrm{~Hz}$ ) and the wheel hop or hub mode (around $14 \mathrm{~Hz}$ ). Also shown in the figure is the response where series stiffness is very large such that it can be treated as a rigid link. There is significant difference in the response at the wheel hub frequency; the resonance is shifted and amplitudes are different. The vehicle body response also shows a similar pattern of behaviour (Figure 2(b)); the
TABLE 1: Parameter values used for the quarter car model.

\begin{tabular}{lc}
\hline System parameter & Parameter value \\
\hline Vehicle parameters & \\
Tyre stiffness $(\mathrm{N} / \mathrm{m})$ & $2 e 5$ \\
Suspension stiffness $(\mathrm{N} / \mathrm{m})$ & $3 e 4$ \\
Suspension damping coefficient (Ns/m) & Varying \\
Hub mass, front $(\mathrm{kg})$ & 40 \\
Quarter of a vehicle body mass $(\mathrm{kg})$ & 250 \\
Stiffness in series $(\mathrm{N} / \mathrm{m})$ & $2 e 5$ \\
\hline
\end{tabular}

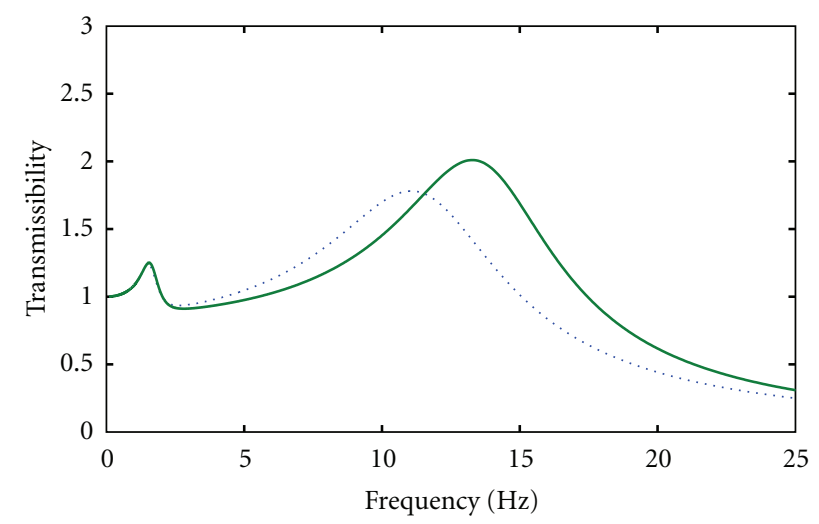

(a)

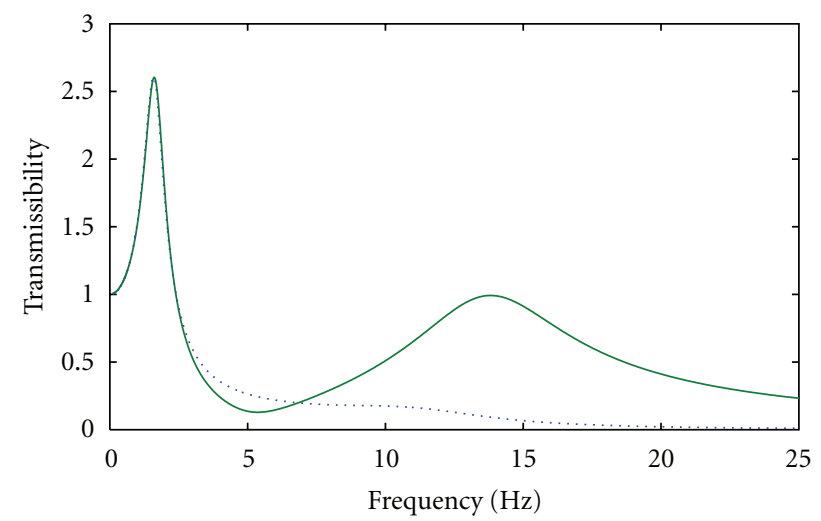

(b)

FIGURE 2: (a) Wheel hub response comparison and (b) vehicle body response comparison (damping coefficient is $1500 \mathrm{Ns} / \mathrm{m}),(\ldots)$ : ordinary model, (-): Maxwell's suspension model.

difference in wheel hub mode contribution to the response is more pronounced. The change in frequency and amplitude, as observed for the model with series stiffness, can have significant influence on the vehicle comfort.

The shift in resonance frequency and the change in amplitude are complex functions of series stiffness, suspension damping coefficient, and suspension stiffness. For complete analysis, forced responses may have to be calculated for all combinations of these parameters. The process can be expensive and may not provide insight into the problem. The aim is to find a combination resulting in large damping ratios and small shift in resonance frequency, without having 
to perform numerous forced vibration analyses. In the next section, a state space representation is explored to find complex eigenvalues from which natural frequencies and damping ratios can be extracted.

\section{Natural Frequencies and Modal Damping Ratios}

Equations of motion can be rearranged for state space formulation as given below:

$$
\begin{gathered}
\ddot{x}_{3}=-\frac{k_{d}}{m_{v}} x_{3}+\frac{k_{d}}{m_{v}} x_{2}, \\
\dot{x}_{2}=\dot{x}_{1}-\frac{k_{d}+k_{s}}{c_{s}} x_{2}+\frac{k_{s}}{c_{s}} x_{1}+\frac{k_{d}}{c_{s}} x_{3} .
\end{gathered}
$$

The equation of motion for $m_{u}$ contains velocity of connection point (Figure 1) between the suspension spring-damper combination and the series stiffness. In the present form of (3), it prohibits straightforward calculation of eigenvalues. To overcome the difficulty, as the force transmitted is same through the suspension spring-damper combination and the series stiffness, term corresponding to the suspension springdamper in (3) is replaced by that of series stiffness; therefore, for free vibration analysis the equation becomes

$$
\begin{aligned}
& m_{u} \ddot{x}_{1}+k_{d}\left(x_{2}-x_{3}\right)+k_{t} x_{1}=0 \\
& \Longrightarrow \ddot{x}_{1}=-\frac{k_{t}}{m_{u}} x_{1}-\frac{k_{d}}{m_{u}} x_{2}+\frac{k_{d}}{m_{u}} x_{3} .
\end{aligned}
$$

Let $y_{1}=x_{1}, y_{2}=\dot{x}_{1}, y_{3}=x_{2}, y_{4}=x_{3}$, and $y_{5}=\dot{x}_{3}$. Therefore,

$$
\mathbf{y}=\left\{\begin{array}{l}
y_{1} \\
y_{2} \\
y_{3} \\
y_{4} \\
y_{5}
\end{array}\right\}=\left\{\begin{array}{l}
x_{1} \\
\dot{x}_{1} \\
x_{2} \\
x_{3} \\
\dot{x}_{3}
\end{array}\right\}, \quad \dot{\mathbf{y}}=\left\{\begin{array}{l}
\dot{y}_{1} \\
\dot{y}_{2} \\
\dot{y}_{3} \\
\dot{y}_{4} \\
\dot{y}_{5}
\end{array}\right\} .
$$

In the state space form, equations of motion are written as

$$
\dot{\mathbf{y}}=\mathrm{Ay}
$$

where

$$
\mathbf{A}=\left[\begin{array}{ccccc}
0 & 1 & 0 & 0 & 0 \\
-\frac{k_{t}}{m_{u}} & 0 & -\frac{k_{d}}{m_{u}} & \frac{k_{d}}{m_{u}} & 0 \\
\frac{k_{s}}{c_{s}} & 1 & -\frac{k_{s}+k_{d}}{c_{s}} & \frac{k_{d}}{c_{s}} & 0 \\
0 & 0 & 0 & 0 & 1 \\
0 & 0 & \frac{k_{d}}{m_{v}} & -\frac{k_{d}}{m_{v}} & 0
\end{array}\right]
$$

Natural frequencies and damping ratios of the system can be obtained by solving eigenvalue problem $\operatorname{det}(\mathbf{A}-\mathbf{I} \lambda)$. The resulting eigenvalues are such that

$$
\lambda_{b}=-\zeta_{b} \omega_{b} \pm j \omega_{d b} ; \quad \lambda_{h}=-\zeta_{h} \omega_{h} \pm j \omega_{d h},
$$

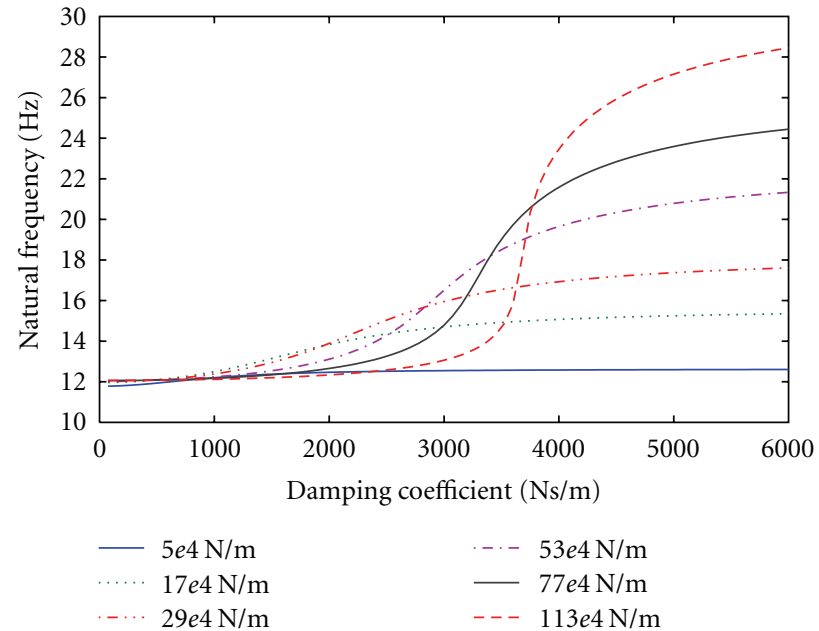

FIGURE 3: Wheel hub natural frequency variation as a function of the suspension damping coefficient and the series stiffness.

where $\lambda_{b}$ is the vehicle body eigenvalue, $\omega_{d b}$ is the damped vehicle natural frequency, $\omega_{b}$ is the vehicle body natural frequency, $\zeta_{b}$ is the vehicle body modal damping ratio, $\lambda_{h}$ is the wheel hop or hub eigenvalue, $\omega_{d h}$ is the wheel hop or hub damped natural frequency, $\omega_{h}$ is the wheel hop or hub natural frequency, and $\zeta_{h}$ is the wheel hop or hub modal damping ratio.

Parametric studies were carried out to find variation of damping ratios and natural frequencies. The base data listed in Table 1 is used. Figure 3 shows variation of wheel hub frequency as a function of the suspension damping coefficient and the series stiffness. There is very little spread at low suspension damping values; in the extreme case of no damping, first element of the stiffness matrix (7) tends to $k_{t}+k_{d} k_{s} /\left(k_{d}+k_{s}\right)$, which for large-series stiffness becomes $k_{t}+k_{s}$. As the suspension damping coefficient increases, so does the frequency spread. For very large values of suspension damping coefficient, first element of the stiffness matrix now tends to $k_{t}+k_{d}$, resulting in an increase in the natural frequency. The increase is gradual for lower series stiffness. In contrast, for larger series stiffness, the transition from lower to higher natural frequency occurs within a small range of suspension damping coefficients. Ideally, in a good design the change in natural frequency should be minimal.

The wheel hub modal damping ratio (Figure 4) variation as a function of suspension damping coefficient and series stiffness is more pronounced than corresponding natural frequency. There is a clear maximum damping ratio for each of the series stiffnesses, increasing as the stiffness increases. The variation in damping ratio is smooth for lower series stiffness; detrimentally the maximum damping ratio reached can be practically very small. For the values of practical importance (series stiffness in the range of $1 \times 10^{5} \mathrm{~N} / \mathrm{m}$ ), the maximum damping ratio achieved can be as low as about 0.2 , which can result in large response amplitude on the vehicle body due to wheel hub mode contribution. The sharp decrease in the damping ratio after reaching the maximum for larger stiffness is due to sudden increase in 


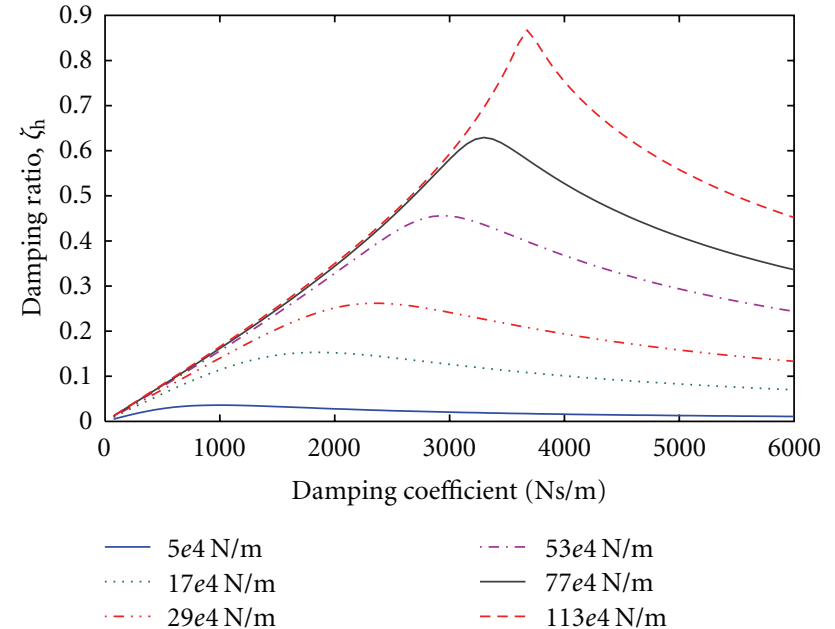

FIGURE 4: Wheel hub modal damping ratio variation as a function of the suspension damping coefficient and the series stiffness.

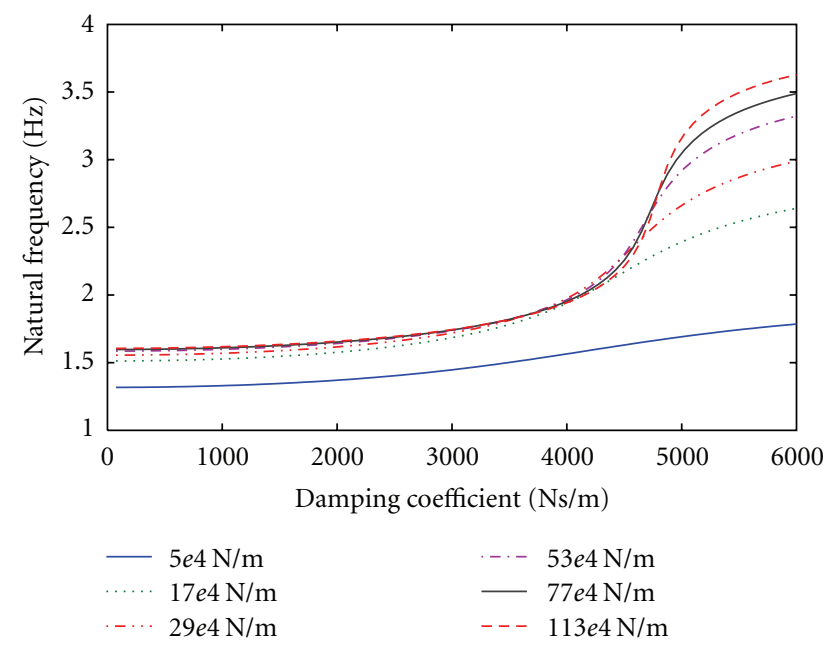

FIGURE 5: Vehicle body mode natural frequency variation as a function of the suspension damping coefficient and the series stiffness.

the natural frequency (Figure 3); it is inversely proportional to the natural frequency.

Figure 5 shows vehicle body frequency variation as a function of the suspension damping coefficient and the series stiffness. For lower suspension damping values, the suspension stiffness and the series stiffness combined result in equivalent stiffness of $k_{d} k_{s} /\left(k_{d}+k_{s}\right)$, which tends to the suspension stiffness $k_{s}$ for large-series stiffness. Hence, there is a spread of frequencies, which is in contrast to the frequencies of the wheel hub mode. As the damping coefficient increases, the effective stiffness tends to $k_{d}$. The natural frequency values show a jump from the lower to the higher extreme for large-series stiffness.

Vehicle body mode damping ratios (Figure 6) are larger than the hub mode damping ratios for smaller series stiffnesses. For larger stiffnesses, the maximum value of damping ratios is reached for similar values of suspension

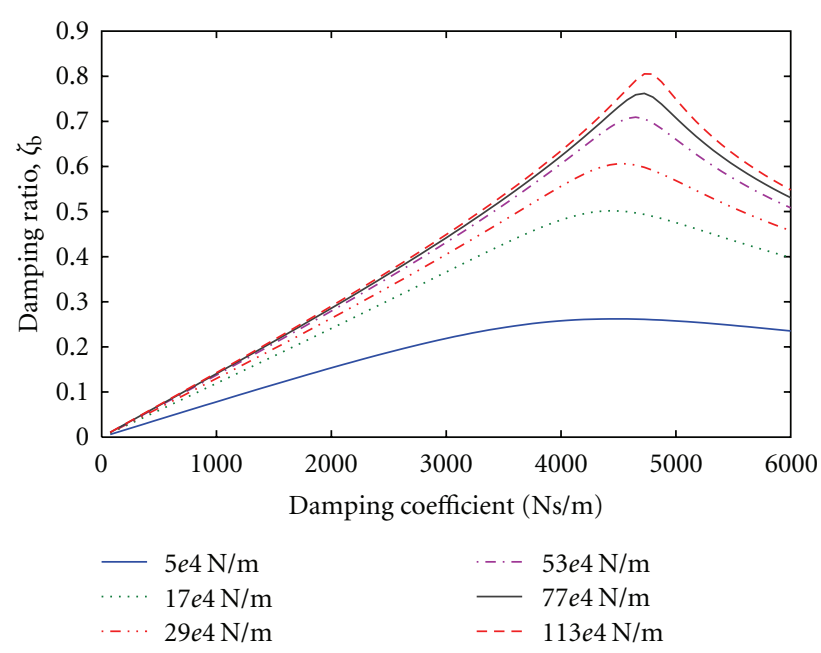

FIGURE 6: Vehicle body modal damping ratio variation as a function of the suspension damping coefficient and the series stiffness.

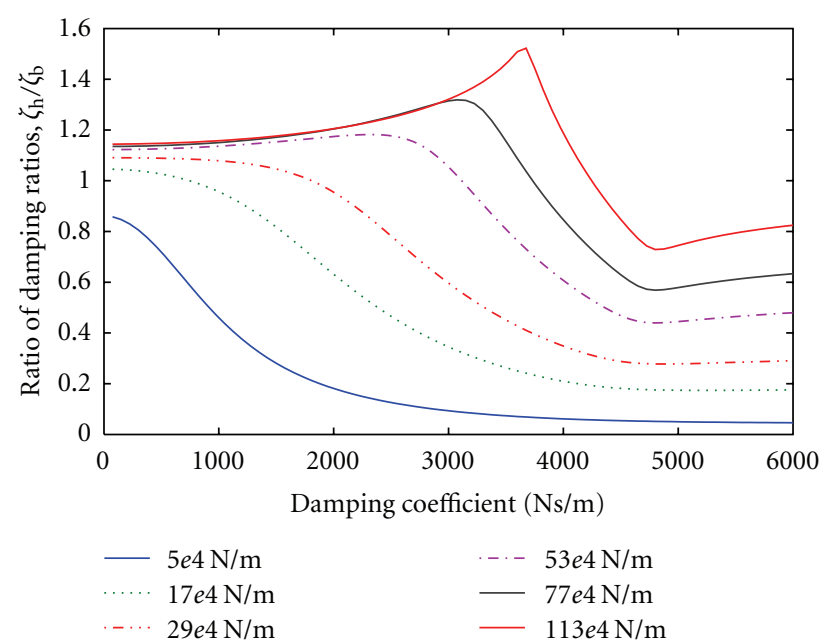

FIGURE 7: Ratio of damping ratios varying as a function of the suspension damping coefficient and the series stiffness.

damping coefficients unlike wider range observed for the wheel hub mode (compare Figures 6 and 4). In addition, the maximum values are reached at much larger suspension damping coefficients, and hence the drop in damping ratio for a further increase may not be of practical significance.

Figure 7 shows the ratio of modal damping ratios; only for a small range of series stiffness values, the ratio remains constant for a limited range of the suspension damping coefficients. Hence for the majority of parameter values, a compromise has to be reached to have suitable damping ratios for both modes. Comparison of Figures 4 and 6 shows the criticalness of wheel hub modal damping. The suspension damping coefficient giving maximum modal damping ratio is a key performance parameter. In the next section, a simple expression is developed to estimate this value based on the analysis of the effective damping coefficient. 


\section{Optimal Damping Coefficient of the Hub Mode}

The effective damping relating to hub mode (see (6)) shows two clearly defined trends: (a) for lower values of suspension damping coefficient it tends to $k_{d}^{2} c_{s} /\left(k_{d}+k_{s}\right)^{2}$, which for large series stiffness value gives $c_{s}$ and (b) for larger suspension damping coefficients it tends to $k_{d}^{2} / c_{s} \omega^{2}$. As the hub mode frequency is a complex function of various parameters, it is difficult to evaluate the latter trend. An appropriate approximation could be based on the fact that the interest is about variation around the wheel hub frequency. For typical vehicle applications, this frequency varies between 12 to $18 \mathrm{~Hz}$; the midpoint value can be suitably assumed to find an estimate of optimum damping coefficient.

Before developing an expression for optimum damping coefficient, the effect of assuming a constant hub mode frequency is analysed. Figure 8 shows variation of effective damping coefficient (first element in the damping matrix, represented here as $c_{\text {eff }}$, of (6)) as a function of suspension damping coefficient. The circular frequency is held constant at $94.25 \mathrm{rad} / \mathrm{s}(15 \mathrm{~Hz})$. Also shown are the values based on exact calculation. The approximation is reasonable representation for up to about $3500 \mathrm{Ns} / \mathrm{m}$, which is a very large damping coefficient for the vehicle suspension systems. The assumption of constant hub mode frequency in calculating optimum damping value, therefore, should not result in significant errors.

The maximum effective damping coefficient leading to an optimal modal damping ratio can be obtained by the following process:

$$
\begin{aligned}
& \frac{d c_{\mathrm{eff}}}{d c_{s}}=\frac{d\left(k_{d}^{2} c_{s} /\left(\left(k_{d}+k_{s}\right)^{2}+c_{s}^{2} \omega^{2}\right)\right)}{d c_{s}}=0 \\
& \Longrightarrow k_{d}^{2}\left(\left(k_{d}+k_{s}\right)^{2}+c_{s}^{2} \omega^{2}\right)-2 k_{d}^{2} c_{s}^{2} \omega^{2}=0 \\
& \Longrightarrow c_{s}=\frac{k_{d}+k_{s}}{\omega} .
\end{aligned}
$$

The result of (14) is used in estimating the optimum effective damping coefficient with hub mode frequency held constant at $15 \mathrm{~Hz}$. Figure 9 shows the variation of the optimal damping coefficient for a given series stiffness. For most practical purposes (series stiffness of the order of tyre stiffness ranging from $0.5 \times 10^{5}$ to $2 \times 10^{5} \mathrm{~N} / \mathrm{m}$ ), the estimates are in reasonable agreement. Equation (14), therefore, can be used to obtain a suitable value of suspension damping coefficient that reduces the response of the vehicle body due to contribution of the wheel hub mode.

\section{Conclusions}

A vehicle quarter car suspension model was refined to include the effect of series stiffness. A novel form of state space equations was used to calculate the natural frequencies and the modal damping ratios. The effect of the suspension

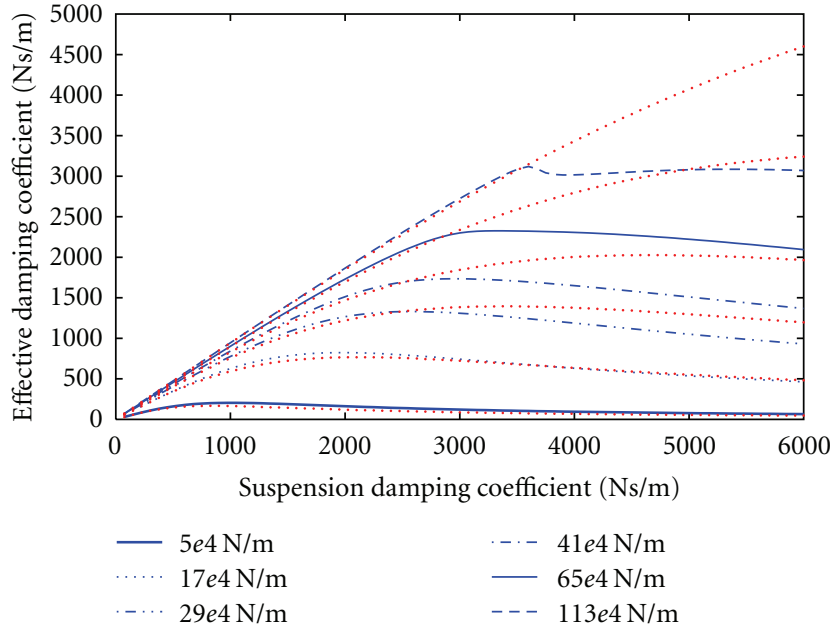

FIGURE 8: Effective damping coefficient variation as a function of the suspension damping coefficient and the series stiffness (...): approximate effective damping coefficient for the hub mode with frequency $15 \mathrm{~Hz}$. Other lines are exact calculations based on the natural frequencies.

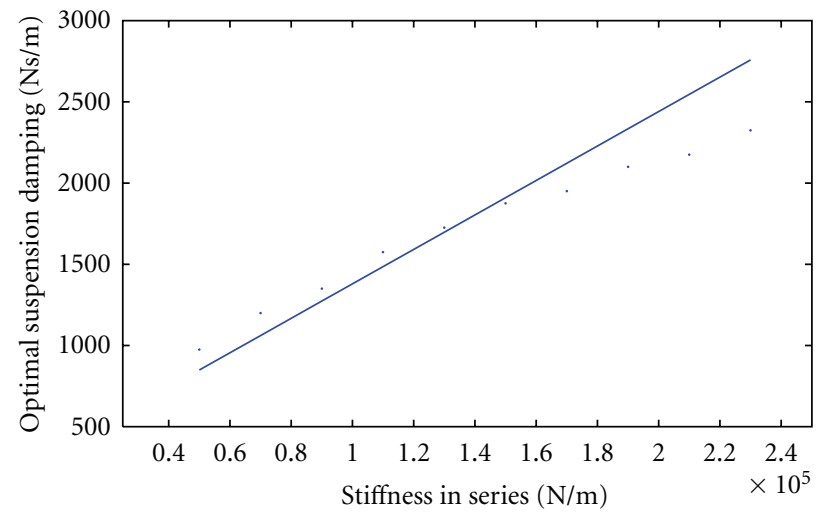

FIGURE 9: Optimal suspension damping coefficient variation as a function of the series stiffness (...): calculated based on the data from Figure 4 and (-): calculated based on (14).

damping and series stiffness was analysed, showing regions of reduced vibration response.

The inclusion of series stiffness reduces the effective damping; the damping ratios achieved at two modes of the quarter car model are smaller than those based on the Kelvin-Voigt model. The variation of the damping ratios is a nonlinear function of suspension damping coefficient and stiffness. In extreme cases, for larger suspension damping coefficients the resulting damping ratios could be negligibly small. The effect may not be equal on the modes of vibration.

The most significant effect of series stiffness was on the wheel hop frequency and the amplitude. Increase in damping beyond the optimal values increases the amplitude at resonance, having a negative impact on vehicle ride comfort. A simplified expression for the optimal suspension damping coefficient was developed eliminating the need for trial simulations. Overall, the model clearly shows 
the influence of series stiffness on the modal damping ratios, the natural frequencies, and hence the dynamic response.

\section{References}

[1] T. D. Gillespie, Fundamentals of Vehicle Dynamics, Society of Automotive Engineers, Warrendale, Pa, USA, 1992.

[2] S. Duym, "An alternative force state map for shock absorbers," Proceedings of the Institution of Mechanical Engineers D, vol. 211, no. 3, pp. 175-179, 1997.

[3] A. Simms and D. Crolla, The Influence of Damper Properties on Vehicle Dynamic Behaviour, SAE, Warrendale, Pa, USA, 2002.

[4] M. Bouazara, M. J. Richard, and S. Rakheja, "Safety and comfort analysis of a $3-\mathrm{D}$ vehicle model with optimal nonlinear active seat suspension," Journal of Terramechanics, vol. 43, no. 2, pp. 97-118, 2006.

[5] M. Bouazara and M. J. Richard, "An optimization method designed to improve 3-D vehicle comfort and road holding capability through the use of active and semi-active suspensions," European Journal of Mechanics, vol. 20, no. 3, pp. 509520, 2001.

[6] M. J. Griffin, "Discomfort from feeling vehicle vibration," Vehicle System Dynamics, vol. 45, no. 7-8, pp. 679-698, 2007.

[7] J. Reimpell, H. Stoll, and J. W. Betzler, The Automotive Chassis: Engineering Principles, Butterworth-Heinman, Woburn, Mass, USA, 2nd edition, 2001.

[8] A. N. Thite, S. Banvidi, T. Ibicek, and L. Bennett, "Suspension parameter estimation in the frequency domain using a matrix inversion approach," Vehicle System Dynamics, vol. 49, no. 12, pp. 1803-1822, 2011. 

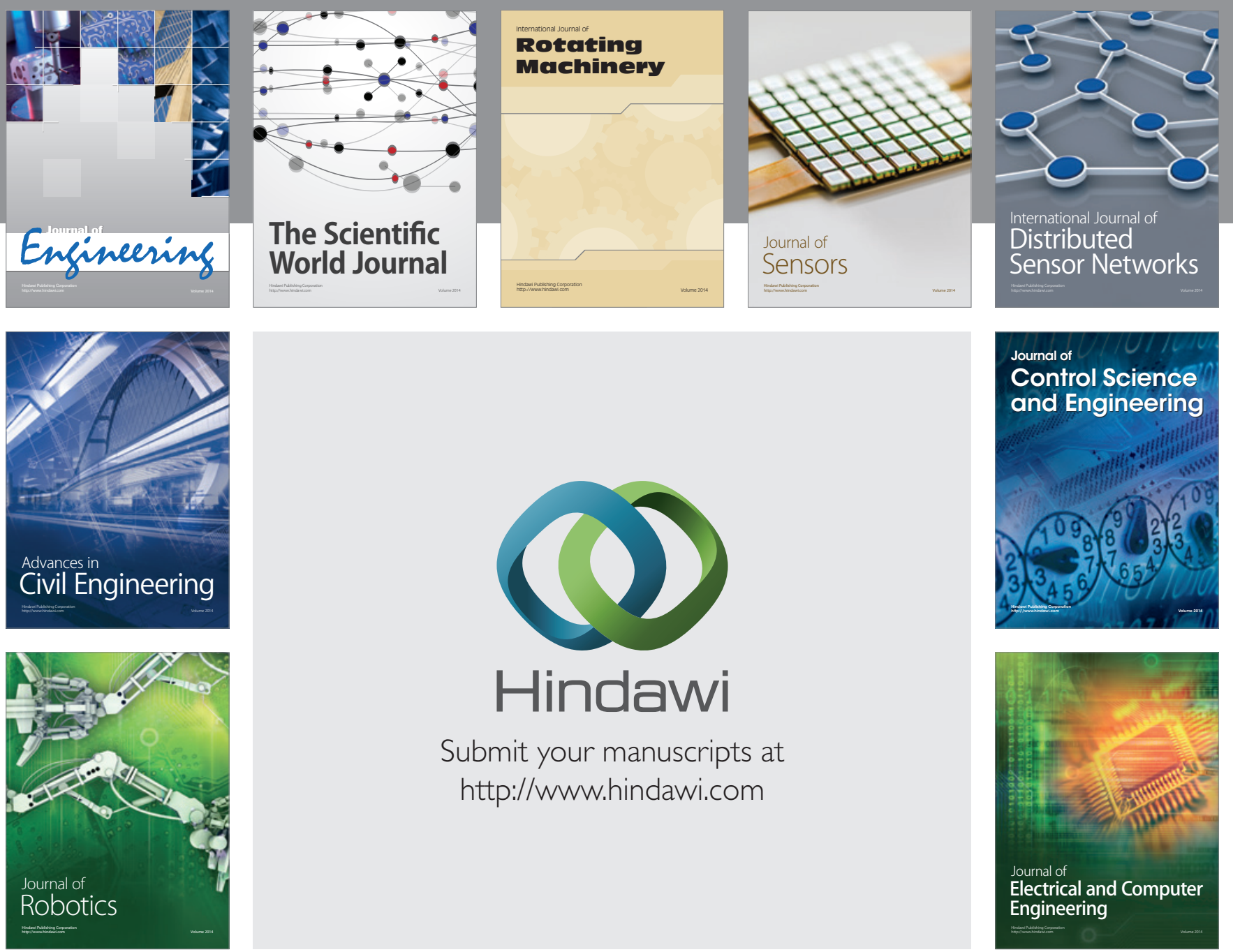

Submit your manuscripts at

http://www.hindawi.com
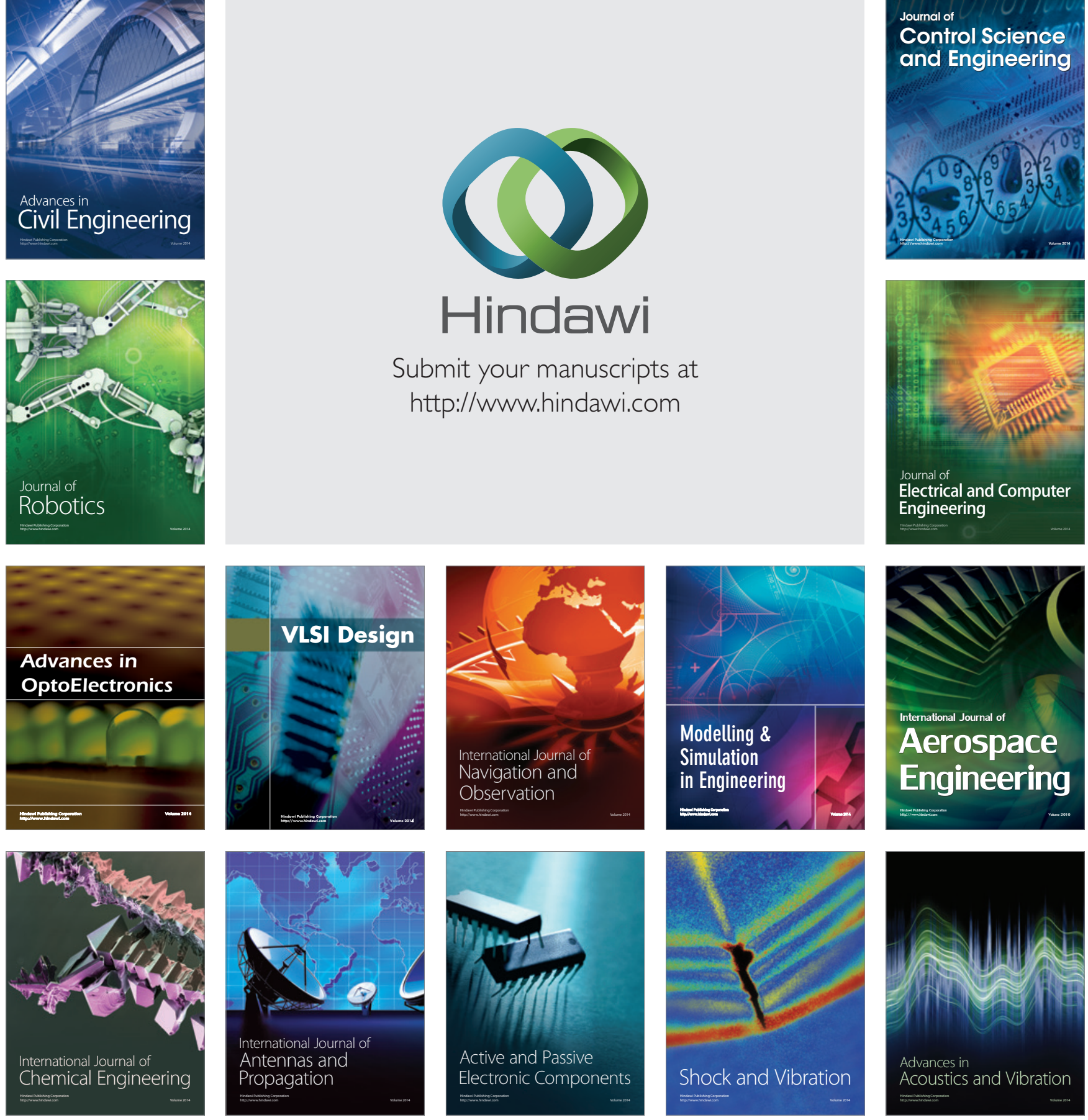